\title{
Comorbidities and risk factors for COVID-19 in a group of Iraqi patients confirmed by real-time PCR test
}

\author{
Z. F. Abbas*1, F. S. Manhal ${ }^{2}$ \\ ${ }^{1 \& 2}$ College of Health and Medical Technologies, Uruk University, Baghdad, Iraq. \\ alialjeboory@yahoo.com
}

\begin{abstract}
The aim of the study was to identify characteristic risk factors predisposing to coronavirus disease 2019 (COVID-19) outbreak in a group of Iraqi patients in Baghdad City. In this study, we included a total of 13411 persons who had been tested for COVID-19, supervised by Al-Razi Medical Center in Baghdad City during the period from June 20 to November 13, 2020. Cases were tested and divided into two groups according to the result of real-time RT-PCR test for COVID-19. A total of 1781 positive cases were detected with confirmed COVID-19. Comorbidities in the study patients were identified as hypertension, diabetes mellitus and respiratory disorders. Hypertension was the most common comorbidity in COVID-19 patients followed by diabetes mellitus. The results of this study showed that patients with comorbidities were significantly associated with positive RT-PCR results related to COVID-19. It was concluded that understanding the comorbidities enhance defining patients with COVID-19 at higher risk. Further research is needed to confirm and clarify the impact of the association of co-morbidities that accompany COVID-19.
\end{abstract}

Crossref

10.36371/port.2021.1.1

Keywords: COVID-19, Comorbidities, Risk Factors, Real-time PCR.

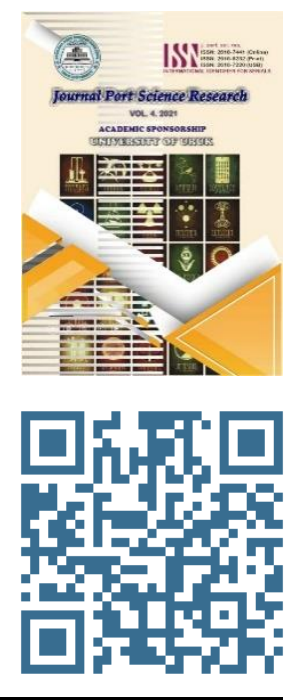

\section{INTRODUCTION}

A new type of coronavirus was identified in December 2019 called novel coronavirus (2019-nCoV, or COVID-19) in Wuhan, China. After that, a rapid spread of this virus was recorded to all parts of China and the world. It can cause symptoms including fever, difficulty breathing, coughing, and invasive lesions in both lungs of patients [1].

In some cases, no symptoms typical of COVID-19 were observed from confirmed PCR testing for Covid19, or the first sign of illness was something other than typical symptoms .[2]

In March 11, 2020, the WHO announced COVID-19 as a pandemic [3]. The disease is highly contagious and each infected person could infect at least 3 other people on average [4]. In symptomatic patients, the clinical manifestations of the disease usually start after less than a week, consisting of fever (body temperature 37 to $38^{\circ} \mathrm{C}$ ), cough, nasal congestion, and fatigue .[5]

Coronavirus Disease 2019 (COVID-19) is a viral respiratory disease caused by the 2019 novel coronavirus (2019-nCoV), which has caused the pneumonia epidemic in the world [6]. This virus has become a global pandemic that has affected the lives of billions of individuals.

SARS-CoV-2 has infected humans in all age groups, of all ethnicities, both males and females while spreading through communities at an alarming rate. Given the nature of this virus, there is much still to be learned; however, we know that the clinical manifestations range from a common cold to more severe diseases such as bronchitis, pneumonia, severe acute respiratory distress syndrome (ARDS), multi-organ failure, and even death.[7]

As the novel coronavirus continues to evolve, there are still many limitations to our knowledge of who exactly this virus would impact critically. Older adults and people of any age who have underlying medical conditions, such as hypertension and diabetes, have shown worse prognosis [8].

Diabetic patients have increased morbidity and mortality rates and have been linked to more hospitalization and intensive care unit (ICU) admissions (Javanbakht, 2021).

People with chronic obstructive pulmonary disease (COPD) or any respiratory illnesses are also at higher risk for severe illness from COVID-19 (Wang, 2020). The risk of contracting COVID-19 in patients with COPD is found to be 4-fold higher than patients without COPD .[9]

Hypertension is the most common comorbidity among COVID-19 patients which is accompanied by higher risk of infection and worse outcomes and prognosis [10]. Frequently, high blood pressure is observed in elderly patients, so it has been recorded in many cases of COVID-19.

However it remains unclear whether uncontrolled blood pressure is a risk factor for getting infected with COVID-19 and developing severe disease or not .[11] 
Given the rapid spread and high mortality rate of COVID-19, it is absolutely necessary to evaluate the possible risk factors affecting the progression of disease in COVID-19 patients. Previous studies show that COVID-19 patients with comorbidity may lead to a poor prognosis. Identifying the most important risk groups is essential when making decisions anti-2019-nCoV therapy (Wang, 2020).

However, risk factors of developing critical conditions in these patients is still a controversial issue.

To date, there are several systematic reviews being published regarding the effect of comorbidities on prognosis of COVID-19 patients. However, much of the previous data analysis is limited by factors such as incomplete prevalence reporting due to the use of non-peer reviewed data and only using data from China .[12]

The aim of this study is to analyze the characteristics of COVID-19 in a group of Iraqi patients and comorbidities to aid the development of strategies to better manage SARS$\mathrm{CoV}$-2-infected patients and to update our information on the influence of comorbidities on the exacerbation of COVID-19 so identifying the patients at higher risk for critical conditions helps to manage the disaster .

\section{MATERIALS AND METHODS}

\subsection{Subjects}

A total of 13411 suspected COVID-19 patients were included in this study, who presented to Al-Razi Medical Center in Baghdad City during the period from June 20 to November 13, 2020. The history of comorbidities data (hypertension, respiratory disorders and diabetes mellitus) was collected from the individuals. All suspected cases of COVID-19 in this study were diagnosed by specialist physicians depending on the clinical examination and laboratory test results.

\subsection{RNA extraction}

Nasopharyngeal swabs were obtained from all participants by specialist medical staff according to standard procedures. Viral nucleic acids (RNA) were extracted and purified from the clinical specimen by using WizPrep ${ }^{\mathrm{TM}}$ Viral DNA/RNA Mini kit (Wizbiosolutions Inc. Korea) according to the manufacturer's instructions. Briefly, the swab was placed into a microcentrifuge tube containing PBS and incubated with shaking for 15 minutes at room temperature.

A $100 \mu 1$ aliquot of the liquid was used for viral extraction. The second step was proceeded through the following procedures of lysis, binding, washing, and elution .

The eluate then contains viral RNA. The nucleic acids were stored at $-80^{\circ} \mathrm{C}$ until use .

The same extracted product of each specimen was used for all the RT-PCRs.

\subsection{Primers and probes}

Primer and probe sets targeting different gene regions (Internal control, spike [S], and $\mathrm{N}$ ) of SARS-CoV-2 were designed and tested. The probes were predicted to specifically detect SARS-CoV-2 and had no homologies with human, other human pathogenic coronaviruses or microbial genes on BLASTn analysis that would potentially produce falsepositive test results as previously described.

\subsection{COVID-19 real-time RT-PCR assays}

Real-time RT-PCR assays for SARS-CoV-2 RNA detection were performed using Rotor-Gene Q, Qiagen) real-time PCR system (Roche, Basel, Switzerland). Biotechrabbit $\mathrm{GmbH}$ (Berlin, Germany) PCR kit was used for detection the different target. Each $20 \mu \mathrm{l}$ reaction mixture contained $10 \mu \mathrm{l}$ of RT-PCR master mix that include Primer Probe and $10 \mu \mathrm{l}$ of final elution volume of extracted samples. The thermal cycling condition was $10 \mathrm{~min}$ at $55^{\circ} \mathrm{C}$ for reverse transcription, $10 \mathrm{~min}$ at $95^{\circ} \mathrm{C}$ for $\mathrm{PCR}$ initial activation, and 40 cycles of $10 \mathrm{~s}$ at $95^{\circ} \mathrm{C}$ and $60 \mathrm{~s}$ at $58^{\circ} \mathrm{C}$.

\subsection{Statistical analysis}

Fisher's exact test or Chi-squared test were used to compare the performance of the assays. P value for Diabetes was 0.05, for respiratory diseases (COPD) was 0.043, and for hypertension was 0.049 . A P value of $<0.05$ was considered statistically significant. All data were analyzed with GraphPad Prism software (GraphPad Software, Inc.). All variables from the univariate analysis were entered into a forward-stepwise multivariate logistic regression analysis.

\section{3 .RESULTS}

Between June 20 and November 13, 2020, 13411 people were included in this study, of whom 1781 were positive for COVID 19 according to Real time PCR. Positive result was according of amplification curve for each molecular observed. In the majority of real-time PCR methods, a positive-result decision is based on the presence of an exponential amplification curve with a $\mathrm{Ct}$ value (Cycle threshold) below a given threshold, and dependent on the total number of cycles programmed for the test .

The result of our study identified hypertension as the most common comorbidity in COVID-19 patients followed by diabetes and chronic obstructive pulmonary disease COPD. The proportions of hypertension, diabetes and COPD in patients with COVID-19 were $41.4 \%, 28.4 \%$ and $16.9 \%$, respectively. The highest percentage of positive PCR test was observed in the 26-40 age group (41.4\%). (Table 1). From the same table, the highest percentage of diabetes cases was detected in the 41-60 age group (40\%), hypertension cases in the 26-40 age group (35.4\%), and COPD cases in the 61-80 age group $(33.8 \%)$. 
* COPD: Chronic Obstructive Pulmonary Disease

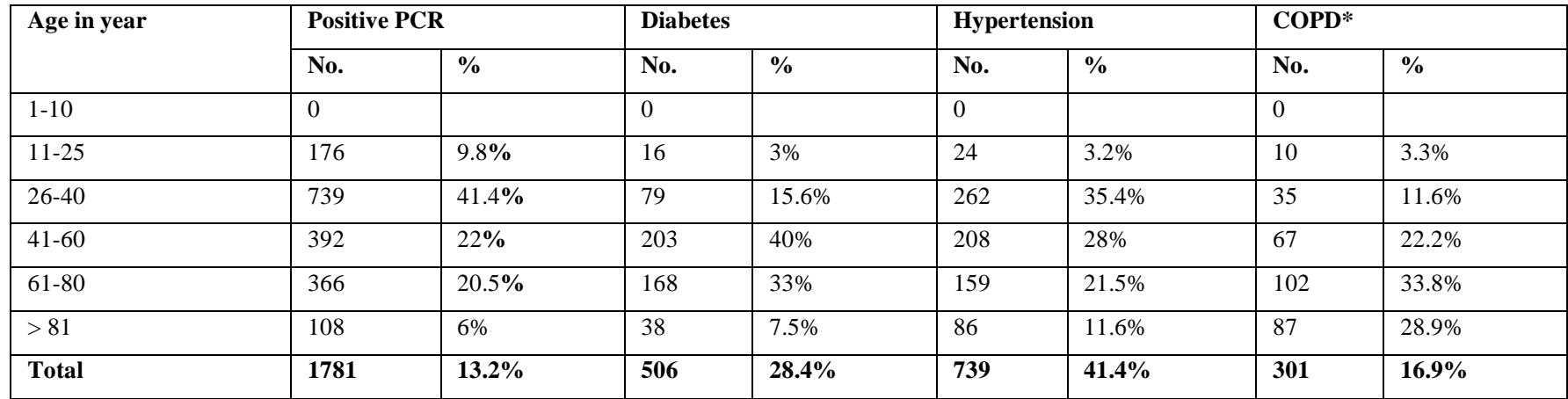

\section{Discussion}

The association between comorbidities and their role in the exacerbation of COVID- 19 in patients leading to death is evaluated in this study. It was clearly demonstrated that $41.4 \%$ of our sturdy patients were detected with hypertension. It was noted by studying and analyzing early data from both China and the United States that hypertension is the most common pre-existing condition among those admitted to hospital, affecting between $30 \%$ and $50 \%$ of patients. Through this observation, we can say that a weak immune system is one of the reasons why people with high blood pressure and other health problems are more susceptible to infection with the Corona virus [13]. From a previous study conducted in Iraq, it was observed that the highest percentage of comorbidities among patients was hypertension (40\%) [14].

There are epidemiological studies presented by Bolin Wang that the presence of common comorbidities increases COVID-19 in patients. However, the relationship between comorbidity and patients with COVID-19 remains unclear (Wang, 2020).

Epidemiological studies have shown that diabetics are more likely to have serious complications from COVID-19 [15]. Our study revealed that $28.4 \%$ of the study patients were diabetics. From a previous study, it was shown that patients with diabetes, compared to those without diabetes, had a $21.8 \%$ higher rate of COVID-19, threefold rates of COVID19 pneumonia, and were more likely to be hospitalized, intubated, admitted to the ICU, and have a fatal outcome [16]. Our study is consistent with the hypothesis that patients with diabetes are more susceptible to infections caused by respiratory viruses such as SARS-CoV-2 virus.

Some scholars believe that the presence of any concurrent illness was more common among patients with severe disease than among those without severe disease (Wang, 2020). The association of severe outcomes in patients with hypertension and diabetes may be partly explained by the increased incidence of thrombotic complications as it has already been shown that patients with hypertension and diabetes have a higher risk of thrombosis [17].

It was observed by Graziano Onder et al that comorbidities such as hypertension, COPD, diabetes, and cardiovascular disease are the most significant risk factors in people when compared to other underlying disease states [18].

It has been found that people with chronic obstructive pulmonary disease (COPD) or any other respiratory disease are more likely to develop severe illness from COVID-19. The risk of contracting COVID-19 in COPD patients was found to be four times higher than in patients without COPD [19]. Our study revealed that $16.9 \%$ of the study patients were detected with COPD. It has been observed through studies that patients with COPD may have a worse outcome from pneumonia caused by COVID-19 compared to patients without COPD [20]. Daniel Meza observed that patients with COPD had higher rates of hospitalization ( $62 \%$ vs $28 \%$ ). The same study detected that mortality rate for patients with COPD was $15 \%$ compared to $4 \%$ in patients without COPD [21].

\section{Conclusion}

The results of our study were to some extent consistent with previous studies regarding the impact of comorbidities that may accompany COVID-19, especially hypertension, diabetes and chronic obstructive pulmonary disease.

The results of this study showed that patients with comorbidities were significantly associated with positive RTPCR results related to COVID-19. It was concluded that understanding the comorbidities enhance defining patients with COVID-19 at higher risk.

Further research is needed to confirm and clarify the impact of the association of co-morbidities that accompany COVID19.

\section{Conflict of interest}

The authors declare that they have no conflict of interest. 


\section{REFERENCES}

[1] Zhu, H., Wei, L., \& Niu, P. (2020). The novel coronavirus outbreak in Wuhan, China. Global Health Research and Policy, 5(1). https://doi.org/10.1186/s41256-020-00135-6

[2] Javanbakht, M., \& Babaee, S. (2021). Tinnitus in COVID-19 patients; A case study. Iranian Journal of War and Public Health, 13(1), 79-83. https://doi.org/10.29252/acadpub.ijwph.13.1.79

[3] Zhu, N., Zhang, D., Wang, W., Li, X., Yang, B., Song, J., ... Tan, W. (2020). A Novel Coronavirus from Patients with Pneumonia in China, 2019. New England Journal of Medicine, 382(8), 727-733. https://doi.org/10.1056/nejmoa2001017

[4] Wu, F., Zhao, S., Yu, B., Chen, Y. M., Wang, W., Song, Z. G., ... Zhang, Y. Z. (2020). A new coronavirus associated with human respiratory disease in China. Nature, 579(7798), 265-269. https://doi.org/10.1038/s41586-020-2008-3

[5] De Almeida-Pititto, B., Dualib, P. M., Zajdenverg, L., Dantas, J. R., De Souza, F. D., Rodacki, M., \& Bertoluci, M. C. (2020). Severity and mortality of COVID 19 in patients with diabetes, hypertension and cardiovascular disease: A metaanalysis. Diabetology and Metabolic Syndrome, 12(1). https://doi.org/10.1186/s13098-020-00586-4

[6] Wang, B., Li, R., Lu, Z., \& Huang, Y. (2020). Does comorbidity increase the risk of patients with COVID-19: evidence from meta-analysis. Aging, 12(7), 6049-6057. https://doi.org/10.18632/aging.103000

[7] Guan, W., Ni, Z., Hu, Y., Liang, W., Ou, C., He, J., ... Zhong, N. (2020). Clinical Characteristics of Coronavirus Disease 2019 in China. New England Journal of Medicine, 382(18), 1708-1720. https://doi.org/10.1056/nejmoa2002032

[8] Guan, W. J., Liang, W. H., Zhao, Y., Liang, H. R., Chen, Z. S., Li, Y. M., Liu, X. Q., Chen, R. C., Tang, C. L., Wang, T., Ou, C. Q., Li, L., Chen, P. Y., Sang, L., Wang, W., Li, J. F., Li, C. C., Ou, L. M., Cheng, B., Xiong, S., ... China Medical Treatment Expert Group for COVID-19 (2020). Comorbidity and its impact on 1590 patients with COVID-19 in China: a nationwide analysis. The European respiratory journal, 55(5), 2000547. https://doi.org/10.1183/13993003.00547-2020

[9] Sanyaolu, A., Okorie, C., Marinkovic, A., Patidar, R., Younis, K., Desai, P., Hosein, Z., Padda, I., Mangat, J., \& Altaf, M. (2020). Comorbidity and its Impact on Patients with COVID-19. SN comprehensive clinical medicine, 2(8), 1069-1076. https://doi.org/10.1007/s42399-020-00363-4

[10] Hosseinzadeh, R., Goharrizi, M. A. S. B., Bahardoust, M., Alvanegh, A. G., Ataee, M. R., Bagheri, M., ... Heiat, M. (2021). Should all patients with hypertension be worried about developing severe coronavirus disease 2019 (COVID-19)? Clinical Hypertension, 27(1). https://doi.org/10.1186/s40885-021-00161-7

[11] Schiffrin, E. L., Flack, J. M., Ito, S., Muntner, P., \& Webb, R. C. (2020, April 29). Hypertension and COVID-19. American Journal of Hypertension. Oxford University Press. https://doi.org/10.1093/ajh/hpaa057

[12] Arman, A., Tajik, M., Nazemipour, M., Ahmadinejad, Z., Shahrestanaki, S. K., Hazrati, E., ... Mansournia, M. A. (2021). Risk factors of developing critical conditions in Iranian patients with COVID-19. Global Epidemiology, 3. https://doi.org/10.1016/j.gloepi.2020.100046

[13] Brunilda Nazario. 2021. Coronavirus and High Blood Pressure: What's the Link? WebMD Medical Reference. https://www.webmd.com/lung/coronavirus-high-blood-pressure\#1. 2021.

[14] Taher, T. M., Sarray, F. T., Farhan Al-Badri, S. A., \& Ghazi, H. F. (2020). Comorbidity and Risk Factors for COVID-19 Confirmed Patients in Wasit Province, IRAQ. AL-Kindy College Medical Journal, 16(supplement), 1-8. https://doi.org/10.47723/kcmj.v16isupplement.174

[15]Zhang, J. Y., Shang, T., Ahn, D., Chen, K., Coté, G., Espinoza, J., ... Klonoff, D. C. (2021). How to Best Protect People With Diabetes From the Impact of SARS-CoV-2: Report of the International COVID-19 and Diabetes Summit. Journal of Diabetes Science and Technology, 15(2), 478-514. https://doi.org/10.1177/1932296820978399

[16]Leon-Abarca, J. A., Portmann-Baracco, A., Bryce-Alberti, M., Ruiz-Sánchez, C., Accinelli, R. A., Soliz, J., \& Gonzales, G. F. (2021). Diabetes increases the risk of COVID-19 in an altitude dependent manner: An analysis of 1,280,806 Mexican patients. PLoS ONE, 16(8 August). https://doi.org/10.1371/journal.pone.0255144

[17] Chen, N., Zhou, M., Dong, X., Qu, J., Gong, F., Han, Y., ... Zhang, L. (2020). Epidemiological and clinical characteristics of 99 cases of 2019 novel coronavirus pneumonia in Wuhan, China: a descriptive study. The Lancet, 395(10223), $507-513$. https://doi.org/10.1016/S0140-6736(20)30211-7 
[18] Onder, G., Rezza, G., \& Brusaferro, S. (2020, May 12). Case-Fatality Rate and Characteristics of Patients Dying in Relation to COVID-19 in Italy. JAMA - Journal of the American Medical Association. American Medical Association. https://doi.org/10.1001/jama.2020.4683

[19] Sanyaolu, A., Okorie, C., Marinkovic, A., Patidar, R., Younis, K., Desai, P., .. Altaf, M. (2020). Comorbidity and its Impact on Patients with COVID-19. SN Comprehensive Clinical Medicine, 2(8), 1069-1076. https://doi.org/10.1007/s42399-020$\underline{00363-4}$

[20] Higham, A., Mathioudakis, A., Vestbo, J., \& Singh, D. (2020). COVID-19 and copd: A narrative review of the basic science and clinical outcomes. European Respiratory Review, 29(158), 1-13. https://doi.org/10.1183/16000617.0199-2020

[21] Meza, D., Khuder, B., Bailey, J. I., Rosenberg, S. R., Kalhan, R., \& Reyfman, P. A. (2021). Mortality from covid-19 in patients with copd: A us study in the n3c data enclave. International Journal of COPD. Dove Medical Press Ltd. https://doi.org/10.2147/COPD.S318000 